\title{
Nephrin and Podocin functions are highly conserved between the zebrafish pronephros and mammalian metanephros
}

\author{
YAYOI FUKUYO ${ }^{1}$, TOMOMI NAKAMURA ${ }^{1,2}$, EKATERINA BUBENSHCHIKOVA ${ }^{1}$, REBECCA POWELL ${ }^{1}$, \\ TAKASHI TSUJI ${ }^{2}$, RALF JANKNECHT ${ }^{1}$ and TOMOKO OBARA ${ }^{1}$ \\ ${ }^{1}$ Department of Cell Biology, University of Oklahoma Health Sciences Center, Oklahoma City, OK 73104, USA; \\ ${ }^{2}$ Department of Biological Science and Technology, Graduate School of Industrial Science and Technology, \\ Tokyo University of Science, Noda, Chiba 278-8510, Japan
}

Received May 4, 2013; Accepted November 22, 2013

DOI: $10.3892 / \mathrm{mmr} .2013 .1844$

\begin{abstract}
The slit diaphragm (SD) is a highly specialized intercellular junction between podocyte foot processes and is crucial in the formation of the filtration barrier in the renal glomeruli. Zebrafish Nephrin and Podocin are important in the formation of the podocyte SD and mutations in NEPHRIN and PODOCIN genes cause human nephrotic syndrome. In the present study, the zebrafish Podocin protein was observed to be predominantly localized in the pronephric glomerular podocytes, as previously reported for Nephrin. To understand the function of Podocin and Nephrin in zebrafish, splice-blocking morpholino antisense oligonucleotides were used. Knockdown of Podocin or Nephrin by this method induced pronephric glomerular hypoplasia with pericardial edema. Human NEPHRIN and PODOCIN mRNA rescued this glomerular phenotype, however, the efficacy of the rescues was greatly reduced when mRNA-encoding human disease-causing NEPHRIN-R1109X and PODOCIN-R138Q were used. Furthermore, an association between zebrafish Nephrin and Podocin proteins was observed. Notably, Podocin-R150Q, corresponding to human PODOCIN-R138Q, markedly interacted with NEPHRIN compared with wild-type PODOCIN, suggesting that this strong binding capacity of mutated PODOCIN impairs the transport of NEPHRIN and PODOCIN out of the endoplasmic reticulum. The results suggest that the functions of Nephrin and Podocin are highly conserved between the zebrafish pronephros and mammalian metanephros. Accordingly, the zebrafish pronephros may provide a useful tool for analyzing disease-causing gene mutations in human kidney disorders.
\end{abstract}

Correspondence to: Professor Tomoko Obara, Department of Cell Biology, University of Oklahoma Health Sciences Center, 975 NE 10th Street, BRC256, Oklahoma City, OK 73104, USA

E-mail: tomoko-obara@ouhsc.edu

Key words: human metanephros, nephrin, nephrotic syndrome, podocin, zebrafish pronephros

\section{Introduction}

The glomerulus is a common structural organization among vertebrates and kidney types (pronephros, mesonephros and metanephros) (1-4). The glomerular filtration barrier, which is responsible for the size and charge-selective properties of the filter, comprises a fenestrated endothelium, composed of the glomerular basement membrane and the podocyte, which are highly specialized epithelial cells of the kidney. The podocyte is composed of three subcellular compartments: a cell body, major processes that extend outward from the cell body and more distally located foot processes that are spanned by a slit diaphragm (SD), which is an essential element of the filtration barrier (5). This basic cellular architecture of podocytes is conserved in vertebrates $(6,8)$.

Intensive research has focused on the molecular basis of SD structure and function and has resulted in the identification of a number of novel SD-associated proteins, including NEPHRIN, NEPH1, CD2-associated protein and PODOCIN (1-5,9-22). These SD-specific proteins are crucial in the formation of the SD and mutations in the NEPHRIN and PODOCIN genes cause congenital nephrotic syndrome of the Finnish type and autosomal recessive steroid-resistant nephrotic syndrome, respectively $(23,25)$. Numerous disease-associated mutations have been reported in the NEPHRIN and PODOCIN genes $(25,26)$, however, the information on the functional abnormalities induced by gene mutations in NEPHRIN and PODOCIN remains limited.

Zebrafish homologues of Nephrin and Podocin are predominantly expressed in the pronephric glomerulus, which is similar to their expression in mammalian metanephric glomerulus and play crucial roles in the formation and function of the SD in the zebrafish pronephric glomerulus $(3,9,27)$. Three morpholino antisense oligos (MOs) used in zebrafish to target Nephrin and Podocin resulted in failure to form normal podocyte architecture, including regular foot processes and SD in zebrafish larvae $(9,22)$. However, updated zebrafish genomic DNA sequences and Gene Tools LLC no longer support these previously used MOs. In the present study, the role of Nephrin and Podocin in the zebrafish pronephros glomerulus was analyzed using different MOs and their evolutionary conservation with human homologues was assessed. 


\section{Materials and methods}

Fish maintenance. The animal experiments were performed in strict accordance with the recommendations in the Guide for the Care and Use of Laboratory Animals of the National Institutes of Health and were approved by the Institutional Animal Care and Use Committee of the University of Oklahoma Health Sciences Center (IACUC protocol no. 12-033 to T.O.). The $\mathrm{AB}$ strain of zebrafish was maintained at $28.5^{\circ} \mathrm{C}$ under a $14 \mathrm{~h}$ light $/ 10 \mathrm{~h}$ dark cycle. Embryos were maintained at $28.5^{\circ} \mathrm{C}$ in $0.5 \mathrm{X}$ E2 egg medium.

Cloning of zebrafish nephrin and podocin. Full-length zebrafish nephrin cDNA that was subcloned into the pCR-BluntII-TOPO vector was a kind gift from Dr Iain Drummond (9). Full-length zebrafish podocin cDNA was obtained by performing RT-PCR on the total RNA isolated from 4 days post-fertilization (dpf) embryos using RNAqueous-4PCR kit (Life Technologies, Carlsbad, CA, USA) and subsequently performing nested PCR. RT-PCR was performed using SuperScript III One-Step RT-PCR System with Platinum Taq High Fidelity (Life Technologies) and nested PCR using Phusion High-Fidelity DNA Polymerase (ThermoScientific, Waltham, MA, USA). The primer sets used were: RT-PCR, forward: 5'-ATC TGC ACT GGC CTC CTG ATA-3' and reverse: 5'-ATG CGA AGG AAA TCC GTC AAC-3' and nested PCR, forward: 5'-CAC CAG AGG ACA CTT CAC AAC A-3' and reverse 5'-CAG CCA ATA ATC AGT ACA GTC TTG AAA-3'. Podocin cDNA was subcloned into pCR-BluntII-TOPO and verified by DNA sequencing.

In situ hybridization. In situ hybridization was conducted as previously described (1-3,9). In brief, the pCR-BluntII-TOPOnephrin and -podocin digested with XhoI were used as a template for anti-sense RNA probe. The probe was synthesized with an SP6 RNA polymerase (New England BioLabs, Ipswich, MA, USA) and DIG-RNA labeling kit (Roche Diagnostics, Mannheim, Germany). Embryos were fixed in $4 \%$ paraformaldehyde (PFA), $0.1 \%$ Tween-20 in PBS for $2 \mathrm{~h}$ at room temperature (RT), altered to $100 \%$ methanol and stored at $-20^{\circ} \mathrm{C}$. Whole-mount in situ hybridization was performed as previously described (28). Following color development, the samples were dehydrated with a graded series of methanol and embedded in JB-4 resin (Polysciences, Inc., Warrington, PA, USA). Ten micron sections were sliced using an RN2255 microtome (Leica Microsystems, Wetzlar, Germany) and counter-stained with special eosin II (BBC Biochemical, Mount Vernon, WA, USA). After mounting in Poly-Mount (Polysciences, Warrington, PA, USA), the stained sections were photographed on a Provis AX-70 microscope (Olympus, Tokyo, Japan) equipped with a RETIGA EXi digital camera (QImaging, Surrey, Canada).

Antibodies. A polyclonal anti-zebrafish Nephrin antibody was prepared as previously described $(3,21)$. Rabbit polyclonal anti-zebrafish Podocin antibody was raised in rabbits using the amino-terminal peptide VKLQEPHKRKE (amino acids 43-53) coupled to KLH. The antiserum was affinity-purified against the immunizing peptide (Covance, Denver, PA, USA).
Immunoblot analysis and immunohistochemistry. Proteins were extracted from 1 to $5 \mathrm{dpf}$ zebrafish. Prior to protein extraction, the yolk ball was removed from $4 \mathrm{dpf}$ zebrafish larvae, as a large amount of yolk-derived proteins occasionally affects SDS-PAGE and immunoblot analysis (29). Deyolked larvae were homogenized and solubilized in the protein extraction buffer (1\% NP-40, $150 \mathrm{mM} \mathrm{NaCl}, 50 \mathrm{mM} \mathrm{KI}, 1 \mathrm{mM}$ EDTA and $10 \%$ glycerol in $50 \mathrm{mM}$ HEPES, pH 7.4). The homogenate was centrifuged at 11,300 x $\mathrm{g}$ for $10 \mathrm{~min}$ and the supernatant was collected as the lysate. Each lysate containing $50 \mu \mathrm{g}$ of protein was subjected to SDS-PAGE (Mini-PROTEAN TGX Gel, 4-15\% gradient; Bio-Rad Laboratories, Hercules, CA, USA) and separated proteins were transferred onto a PVDF membrane (Immobilon Transfer Membrane; Millipore, Billerica, MA, USA). Each membrane was incubated with the primary antibody for $2 \mathrm{~h}$ at RT and then with an anti-HRP-conjugated secondary antibody (working dilution 1:5,000; Jackson ImmunoResearch Laboratories, West Grove, PA, USA) for $1 \mathrm{~h}$ at RT. Dilutions of primary antibodies were 1:2,500 for anti-Nephrin, 1:5,000 for anti-Podocin and 1:5,000 for anti- $\alpha$-tubulin.

Immunohistochemical detection of Nephrin and Podocin. Immunohistochemical analysis was performed as previously described (3). Larvae were fixed with Dent's fixative (20\% DMSO in methanol) overnight at $4^{\circ} \mathrm{C}$. Fixed samples were rehydrated with a graded series of methanol and washed with PBS containing $0.5 \%$ Triton X-100 (PBSTx). For antigen retrieval, the samples were heated in Antigen Retrieval Reagent Universal (R\&D Systems, Minneapolis, MN, USA) for $15 \mathrm{~min}$ at $95^{\circ} \mathrm{C}$ on a heat block. Subsequently, the samples were blocked with the incubation solution (PBSTx containing 10\% normal goat serum and $1 \%$ DMSO) for $2 \mathrm{~h}$ at RT and incubated with the primary antibody (working dilution 1:100) in the incubation solution for $12 \mathrm{~h}$ at $4^{\circ} \mathrm{C}$. After washing with PBSTx, the samples were incubated with Alexa-Fluor546-conjugated goat anti-rabbit IgG (H+L; Jackson ImmunoResearch Laboratories) and diluted with the incubation solution $(1: 1,000)$ for $2 \mathrm{~h}$ at RT. Stained samples were dehydrated with a graded series of methanol, embedded in JB-4 resin (Polysciences, Inc.) and sliced into $10-\mu \mathrm{m}$ sections with a RN2255 microtome. The sections were photographed with an FV-1000 confocal laser scanning microscope (Olympus).

Production of Flag-tagged zebrafish podocin. cDNA corresponding to zebrafish podocin was generated using RT-PCR, digested with BamHI and XbaI, subcloned into pEV3S-Flag vector and verified by DNA sequencing. This cDNA was used to generate the R150Q mutation, which was also cloned into the pEV3S-Flag vector and verified by DNA sequencing. Human embryonic kidney $293 \mathrm{~T}$ cells, which were grown to $25 \%$ confluency in $60-\mathrm{mm}$ dishes, were transiently transfected with $2 \mu \mathrm{g}$ Flag-Podocin and $7 \mu \mathrm{g}$ pBluescript $\mathrm{KS}^{+}$ using the calcium phosphate coprecipitation method (30). Following 12-h incubation, the precipitate was removed by washing twice with $2 \mathrm{ml}$ PBS and the cells were incubated for an additional $36 \mathrm{~h}$ in DMEM supplemented with $10 \%$ fetal bovine serum at $37^{\circ} \mathrm{C}$. Thereafter, the cells were lysed by boiling in Laemmli buffer and the insoluble material was removed by centrifugation (31). The resultant protein lysate 
was subjected to SDS-PAGE and the separated proteins were transferred to a PVDF membrane (Immobilon; Millipore) and processed for immunoblotting with the indicated antibodies (32).

MOs and efficacy checking. MOs were designed for the splice blocking of nephrin-exon 25 (nephrinMOex25: 5'-TGC ACC AAC ACG ACT CAC CTC TGC TC-3') and of podocin-exon 3 (podocinMOex3: 5'-TGC GAT TTA AAA CGT GTA CCA GGG C-3') (Gene Tools LLC, Philomath, OR, USA). The two morpholinos target the splice acceptor site. The morpholinos were diluted with the injection solution containing $100 \mathrm{mM}$ $\mathrm{KCl}$ and $10 \mathrm{mM}$ HEPES (pH 7.6) and were injected at a final concentration of $0.15 \mathrm{mM}$ (nephrinMOex25) and $0.25 \mathrm{mM}$ (podocinMOex3) into one- or two-cell stage embryos using a Nanoliter 2000 microinjector (World Precision Instruments, Sarasota, FL, USA). The injected volume was $4.6 \mathrm{nl}$. Splice blocking was verified using RT-PCR and nested PCR. Total RNA was isolated from five embryos with an RNAqueous-4PCR kit (Life Technologies). RT-PCR was performed using SuperScript III One-Step RT-PCR System with Platinum Taq High Fidelity (Life Technologies) followed by nested PCR using Phusion High-Fidelity DNA Polymerase (New England Biolabs). The primer sets used were: forward, 5'-GGC AGG ATC TGC AAG CTA CAT-3 and 5'-CTC AGG GCC TTC AGG GT GAG-3' (RT-PCR for nephrin); 5'-CTC CTG AAC CCA TTC ATC TGC-3' and 5'-CTC ATA GAC GCT GCT GTC AGG-3' (nested PCR for nephrin); 5'-GAT GCT TCC TGC GGA GAT AGA-3' and 5'-TTC CTG TCC AGC AAA ATG TCA-3' (RT-PCR for podocin) and 5'-TCA TCT CTA GCA GCA CGG TTG-3' and 5'-TCT GGA ATG CTA GCG AAG GAG-3' (nested PCR for podocin). Altered PCR products were subcloned into pCR-BluntII-TOPO and sequenced.

Hematoxylin and eosin staining. Hematoxylin and eosin staining was conducted as previously described (32). In brief, larvae were fixed with histology fixative $(1.5 \%$ glutaraldehyde, $4 \%$ PFA, 3\% sucrose in $0.1 \mathrm{M}$ phosphate buffer, $\mathrm{pH} 7.3$ ) overnight at $4^{\circ} \mathrm{C}$, dehydrated with a graded series of methanol and embedded in JB-4 resin. Four micron sections were sliced and stained with Harris hematoxylin and special eosin II (BBC Biochemical, Mount Vernon, WA, USA). The stained sections were photographed with a Provis AX-70 microscope equipped with a RETIGA EXi digital camera (QImaging).

Transmission electron microscopy. Transmission electron microscopy was conducted as previously described (3). Larvae were fixed with histology fixative overnight at $4{ }^{\circ} \mathrm{C}$. The samples were immersed in $1 \% \mathrm{OsO}_{4}$ in $0.1 \mathrm{M}$ phosphate buffer for $1 \mathrm{~h}$, dehydrated with a graded series of ethanol and then embedded in Epon-Araldite resin (Electron Microscopy Sciences, Hatfield, PA, USA). Ultrathin silver-gold sections were produced with an ultra $45^{\circ}$ diamond knife (Diatome, Biel, Switzerland) and were transferred to copper grids (50 mesh, Nisshin EM) with a carbon-coated Formvar membrane. The sections were stained with uranyl acetate and lead citrate and photographed on a H-7600 transmission electron microscope (Hitachi High Technologies Inc., Schaumburg, IL, USA) equipped with a Kodak $2 \mathrm{~K}$ x $2 \mathrm{~K}$ digital camera.
Co-immunoprecipitation assay. cDNA encoding the intracellular region of zebrafish Nephrin (amino acids 1,065-1,242) was generated by performing PCR on the total RNA from $2 \mathrm{dpf}$ zebrafish larvae as a template, subcloned into $\mathrm{pCS}^{+}-6 \mathrm{Myc}$ vector and verified by DNA sequencing. Generation of Flag-Podocin vector was mentioned earlier. Each $1 \mu \mathrm{g}$ of indicated Flag-Podocin and 6Myc-Nephrin-C vectors were transfected together with $7 \mu \mathrm{g}$ pBluescript $\mathrm{KS}^{+}$into $293 \mathrm{~T}$ cells essentially as previously described (31). Following $36 \mathrm{~h}$ incubation, the cells were lysed in $600 \mu 150 \mathrm{mM}$ Tris (pH 7.4), $50 \mathrm{mM} \mathrm{NaF}, 150 \mathrm{mM} \mathrm{NaCl}, 0.2 \mathrm{mM}$ DTT, 0.5\% NP-40, $1 \mathrm{mM}$ PMSF, $10 \mu \mathrm{g} / \mathrm{ml}$ leupeptin, $2 \mu \mathrm{g} / \mathrm{ml}$ aprotinin, $1 \mu \mathrm{g} / \mathrm{ml}$ pepstatin A and $0.2 \mathrm{mM} \mathrm{Na} \mathrm{VO}_{4}$. Cell lysates were processed as described earlier utilizing the anti-Flag M2 monoclonal antibody (Sigma-Aldrich, St. Louis, MO, USA) for the immunoprecipitation (34). Immunoprecipitated complexes were resolved by SDS-PAGE and co-precipitated proteins were detected by anti-Myc immuno-blot analysis (35).

Synthesis and egg injection of capped RNA. The human NEPHRIN and PODOCIN cDNAs were kind gifts from Dr Lawrence Holzman. PCR was used to introduce a non-sense mutation into human NEPHRIN cDNA causing R1109X and a single base substitution into human PODOCIN cDNA causing $\mathrm{R} 138 \mathrm{Q}$. The following primer sets were used for the generation of mutated cDNA: for NEPHRIN mutant: HNEPHRIN-forward: 5'-GTC AAA GCT TAT GGC CCT GGG GAC GAC GCT CAG and -reverse: 5'-AAG GAT AGC GGC CGC CTA CAC CAG ATG TCC CCT CAG CTC GAA G; HNEPHRIN-1109X-forward: 5'-GGG TCG GAA GAG GAC TAG GTC AGG AAC GAA TAT GAG GAG AGC C-3' and -reverse: 5'-GGC TCT CCT CAT ATT CGT TCC TGA CCT AGT CCT CTT CCG ACC CTG CC-3'; for PODOCIN mutant: HPODOCIN-forward: 5'-GTC AAA GCT TAC CAT GGA GAG GAG GGC GCG GAG CTC CTC CAG-3' and -reverse: 5'-AAG GAT AGC GGC CGC CTA TAA CAT GGG AGA GTC TTT CTT TTT AGG-3', HPODOCIN-138Q-forward: 5'-AGA GTA ATT ATA TTC CAG CTG GGA CAT CTG CTT CCT GGA AGA G-3' and -reverse: 5'-CAG ATG TCC CAG CTG GAA TAT AAT TAC TCT TTC ATA CTC TTG TAC AAC C-3'. The PCR products were subcloned into pCR-BluntII-TOPO and verified by DNA sequencing. These vectors were digested with restriction enzymes and used as templates for the synthesis of capped RNA. Capped RNA was synthesized using the mMESSAGE mMACHINE kit (Life Technologies). Synthesized capped RNA ( $\sim 64$ pg for human wild-type and mutated NEPHRIN and $\sim 32 \mathrm{pg}$ for human wild-type and mutated PODOCIN) was injected into one-cell stage embryos along with morpholinos $(0.15 \mathrm{mM}$ nephrinMOex 25 or $0.25 \mathrm{mM}$ podocinMOex3).

\section{Results}

$m R N A$ and protein expression. Throughout zebrafish pronephric development [ $34 \mathrm{~h}$ post-fertilization (hpf) to $4 \mathrm{dpf}$ ], nephrin and podocin mRNA expression was detected in the glomerular primordia and compared with the glomerulus (Fig. 1A-H). The expression patterns of nephrin and podocin markedly resembled that for Wilms' tumor 1a, which is predominantly expressed in the podocytes within the pronephric glomerulus (1). 
Antibodies specific to Nephrin and to Podocin were developed to determine their expression pattern and localization. The specificity of the anti-Nephrin antibody was previously described (3). To examine the specificity of the anti-Podocin antibody, the possibility of a reaction with Flag-tagged zebrafish Podocin expressed in 293T cells was investigated. Immunoblot analysis showed that the anti-Podocin antibody was capable of detecting an $\sim 55 \mathrm{kDa}$ protein, corresponding to the Flag-tagged Podocin (Fig. 1I). These anti-Nephrin and anti-Podocin antibodies could also detect $\sim 240$ and $\sim 40 \mathrm{kDa}$ protein bands corresponding to endogenous Nephrin and Podocin, respectively (Fig. 1J).

With these antibodies, Nephrin and Podocin were immunohistochemically detected in $4 \mathrm{dpf}$ larvae as two continuous tortuous lines, which were likely to be situated along the glomerular basement membrane (Fig. 1K and L). A similar tortuous linear pattern for Nephrin localization was also observed for the rodent metanephric glomerulus $(36,37)$.

Splice blocking of nephrin and podocin pre-mRNA. To determine the function of Nephrin and Podocin in the development of the zebrafish pronephric glomerulus, splice blocking MOs were designed for Nephrin and Podocin to target the splice acceptor sites of exon 25 for nephrin (nephrinMOex25) and exon 3 for podocin (podocinMOex3), respectively (Fig. 2A and $\mathrm{B})$.

Injection of nephrinMOex 25 resulted in mis-spliced mRNA as detected by an altered RT-PCR product (Fig. 3A). This altered product was $\sim 50$ bp smaller than the wild-type. Sequencing of the altered RT-PCR product revealed a deletion of exon 25 that resulted in a frame shift and non-sense translation of the subsequent exons, resulting in an almost complete loss of the intracellular domain of the Nephrin protein in nephrinMOex25 morphants (Fig. 2A). The efficacy for nephrinMOex 25 was consistently recognized from 1 to $4 \mathrm{dpf}$ (Fig. 3A) and the protein expression of Nephrin was reduced at $4 \mathrm{dpf}$ (Fig. 3C).

Administration of podocinMOex 3 also resulted in mis-spliced mRNA as detected by an altered RT-PCR product that was $\sim 150$ bp smaller than the wild-type amplicon (Fig. 3B). Sequencing of the altered RT-PCR product revealed an in-frame deletion of exons 3 and 4 (Fig. 2B). The truncated RT-PCR product was recognized from 1 to $4 \mathrm{dpf}$, although a significant expression of intact Podocin mRNA was also detected (Fig. 3B). The reduced expression of the Podocin protein was observed in the $3 \mathrm{dpf}$ morphants, however, the expression was recovered at the same level as in the control at $4 \mathrm{dpf}$ (Fig. 3D).

NephrinMOex25 and PodocinMOex3 morphants exhibited pericardial and yolk edema from 3 to $4 \mathrm{dpf}$ (Fig. 2C-E). In addition to edema, the majority of the morphants showed body-axis curvature (Fig. 2C-E). In wild-type zebrafish, a pair of pronephric glomerular primordia merged with glomerular capillaries and mesangium at the midline to form a single glomerulus (Fig. 2F) (1). The glomerulus was smaller in size in the morphants compared with that in the wild-type larvae and contained poorly developed glomerular capillaries and mesangium (Fig. 2G and $\mathrm{H}$ ).

In $4 \mathrm{dpf}$ control larvae, regular foot processes with SD covered a large area of the urinary surface of the glomerular

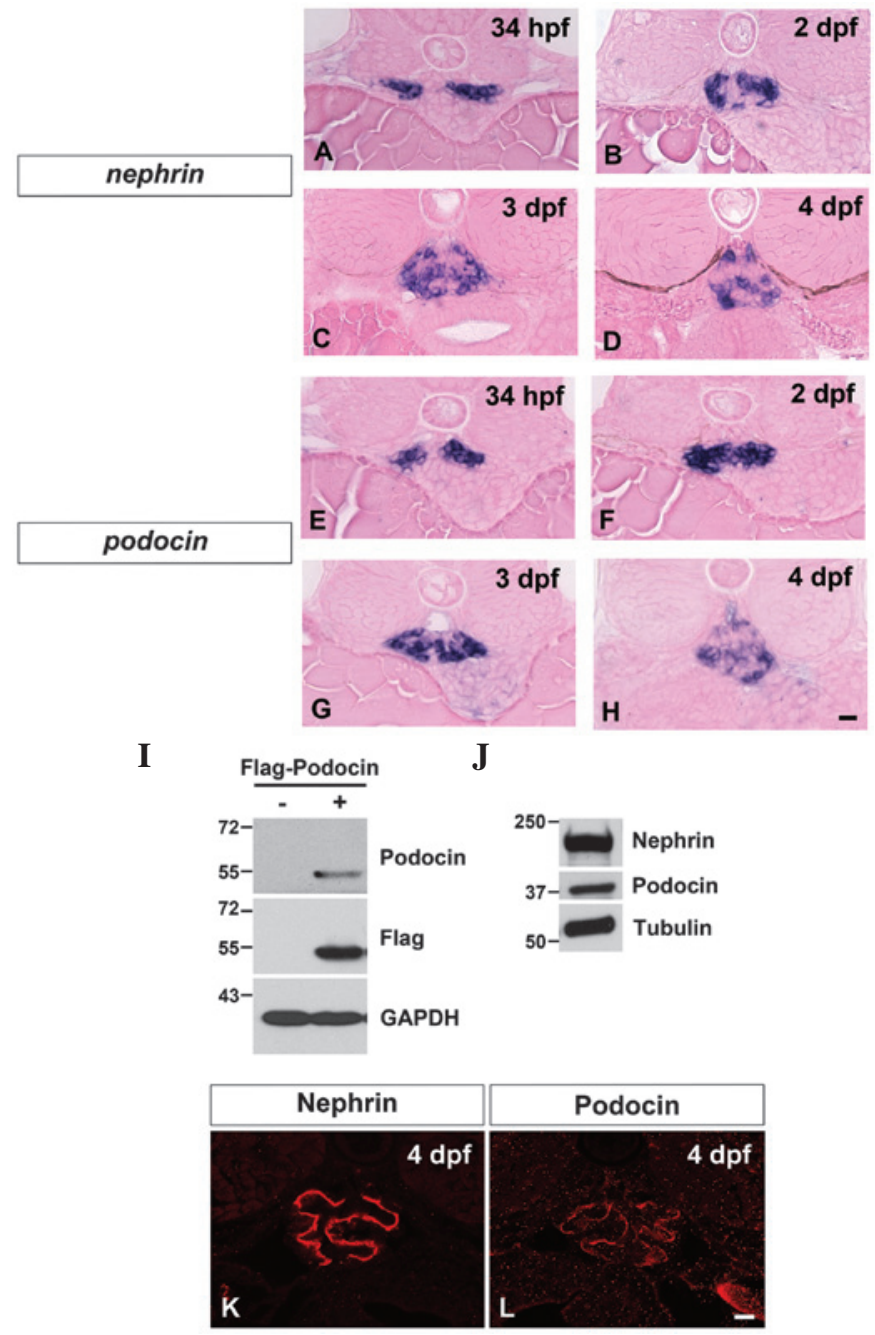

Figure 1. mRNA and protein expression. (A-H) mRNA expression of (A-D) nephrin and (E-H) podocin is already detected in the zebrafish pronephric glomerular primordial at $34 \mathrm{hpf}$ and sustained throughout the following developmental period (2-4 dpf). (I) Specificity of newly-developed anti-zebrafish Podocin antibody. The antibody detects a Flag-tagged full-length zebrafish Podocin. (J) Intrinsically expressed Nephrin and Podocin in $4 \mathrm{dpf}$ zebrafish are detected by immunoblot analysis using the anti-Nephrin and anti-Podocin antibodies. ( $\mathrm{K}$ and $\mathrm{L}$ ) Nephrin and Podocin proteins were detected as a pair of tortuous line segments by immunohistochemistry. (H and L) Bar scales, $10 \mu \mathrm{m}$. dpf, days post-fertilization; hpf, hours post-fertilization.

basement membrane (Fig. 2I). At the same stage, nephrinMOex25 morphants were associated with a great reduction in the area covered with regular foot processes. Instead of the foot processes, irregularly shaped processes covered the larger area of its urinary surface and the SD was not observed between these irregularly shaped processes (Fig. 2J). In 4 dpf podocinMOex 3 morphants, the foot processes with SD covered a large area of the glomerular wall, however, the processes varied in shape and size (Fig. 2K).

Rescue of the morphant phenotypes by human intact mRNA. To investigate whether administration of human intact mRNA rescues the morphant phenotypes, splice blocking morpholino and human wild-type mRNA were simultaneously injected into one-cell stage embryos. Human NEPHRIN and PODOCIN mRNA were detected in the 4 dpf-injected larvae by RT-PCR (Fig. 4B), indicating that the injected mRNA remained until at 
A wild-type nephrin

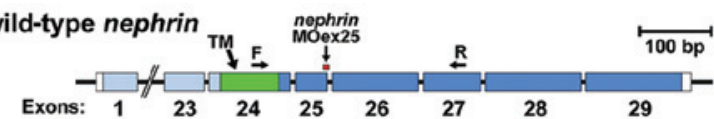
nephrinMOex25



B wild-type podocin

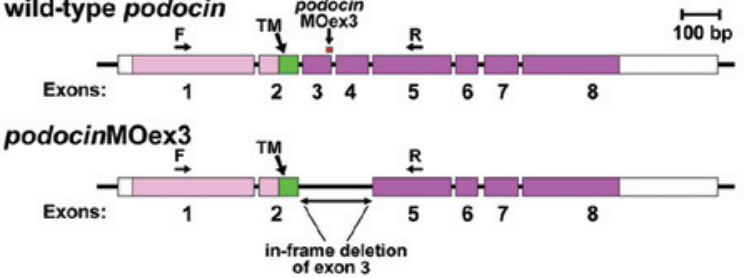

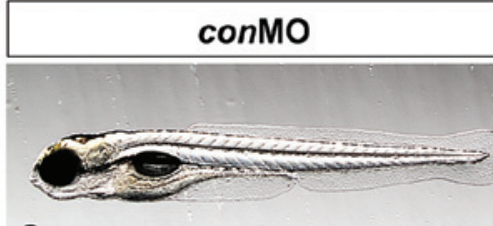

C
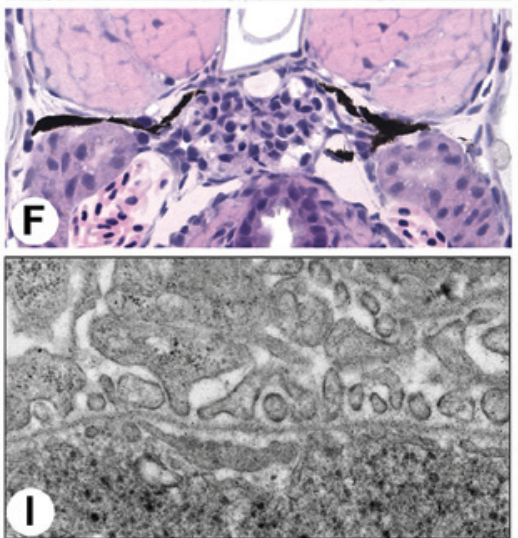
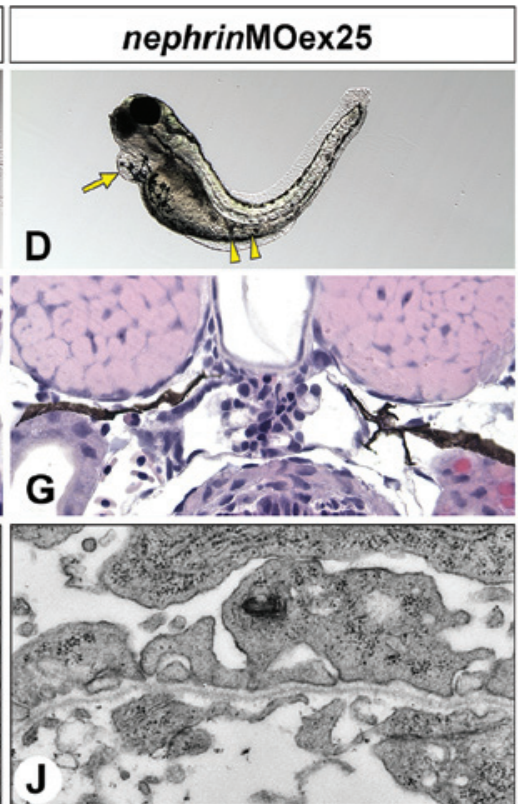

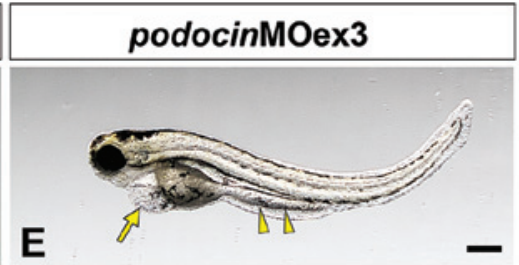

E
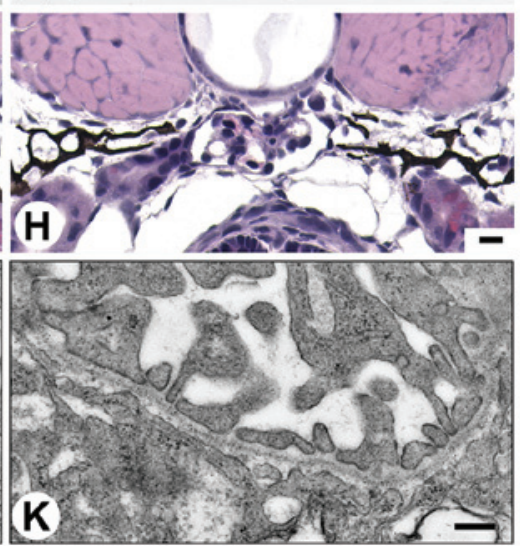

Figure 2. Gene knockdown using splice-blocking morpholinos. (A) nephrinMOex25 targets the splice-acceptor site of nephrin exon 25 and induces a frame-shift deletion. (B) PodocinMOex3 targets the splice-acceptor site of podocin exon 3 and induces an in-frame deletion of exons 3 and 4 . (F) and (R) show the sites of primer sets for the testing of the efficacy of target modification (PCR data shown in Fig. 1A and B). (C-E) Lateral view of 4 dpf larvae. NephrinMOex25 and podocinMOex3 morphants exhibit pericardial edema (arrow), edematous swelling of yolk sac extension (arrowheads) and body-axis curvatures. (F-H) Glomerulus in the 4 dpf larvae in HE-stained sections. The control larva exhibits a well-developed glomerular capillary and mesangium. In the morphants, glomerular development is markedly disrupted. (I-K) Ultrastructure of glomerular capillary wall. The control larva exhibits fine regular foot processes and slit diaphragm, present in a 'beads on a string' pattern. In the nephrinMOex 25 morphant, the regular foot processes are not observed at all, however, in the podocinMOex 3 morphant, foot processes with slit diaphragm are formed. TM, transmembrane domain coding region. Bar scales, $250 \mu \mathrm{m}$ in (E); $10 \mu \mathrm{m}$ in $(\mathrm{H})$ and $50 \mathrm{~nm}$ in $(\mathrm{K})$. dpf, days post-fertilization.

A

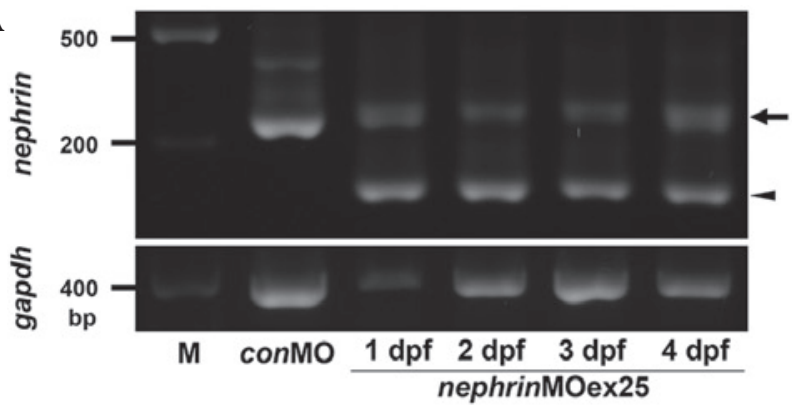

C

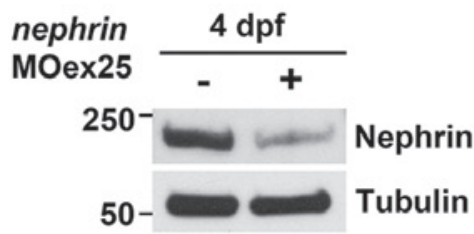

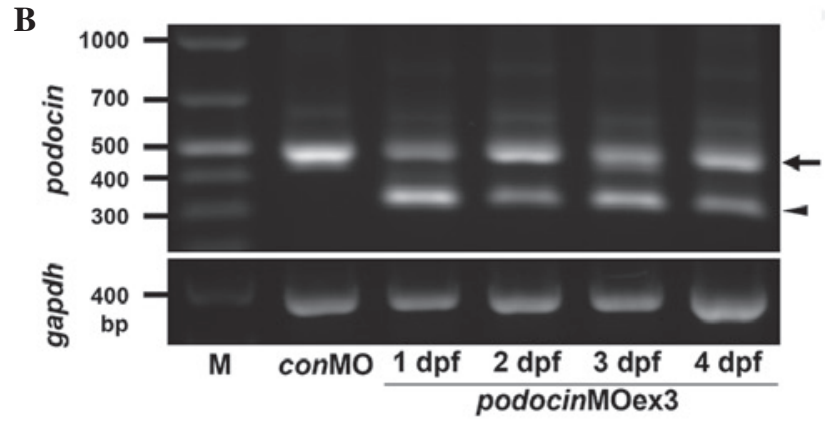

$\mathbf{D}$

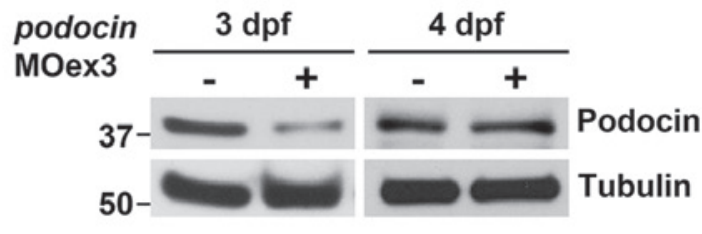

Figure 3. Morpholino efficacy. (A and B) Injection of nephrinMOex25 and podocinMOex3 resulted in the mis-splicing of mRNA as detected by an altered RT-PCR product (arrowheads). Arrows indicate intact mRNA. (A) The efficacy for nephrinMOex 25 is consistent from 1 to 4 dpf. (B) By contrast, the efficacy for podocinMOex 3 is recognized from 1 to $4 \mathrm{dpf}$, although a significant expression of intact podocin mRNA is also detected. (C) In the nephrinMOex 25 morphants, the protein expression of Nephrin was reduced at 4 dpf larvae. (D) In the podocinMOex 3 morphants, a reduced expression of Podocin protein is observed at $3 \mathrm{dpf}$, however, the expression is recovered at the same level of control at $4 \mathrm{dpf}$. dpf, days post-fertilization. 
A

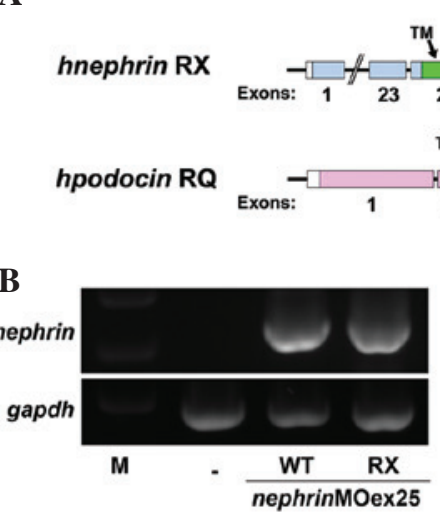

D

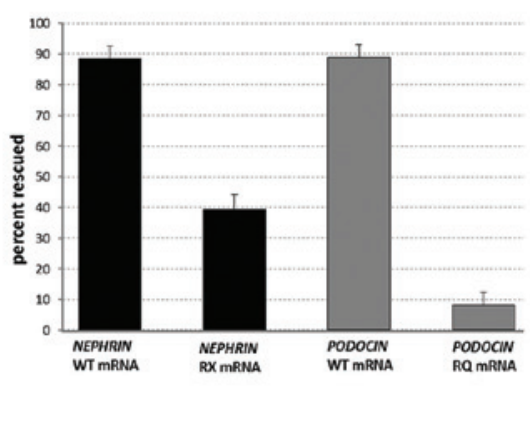

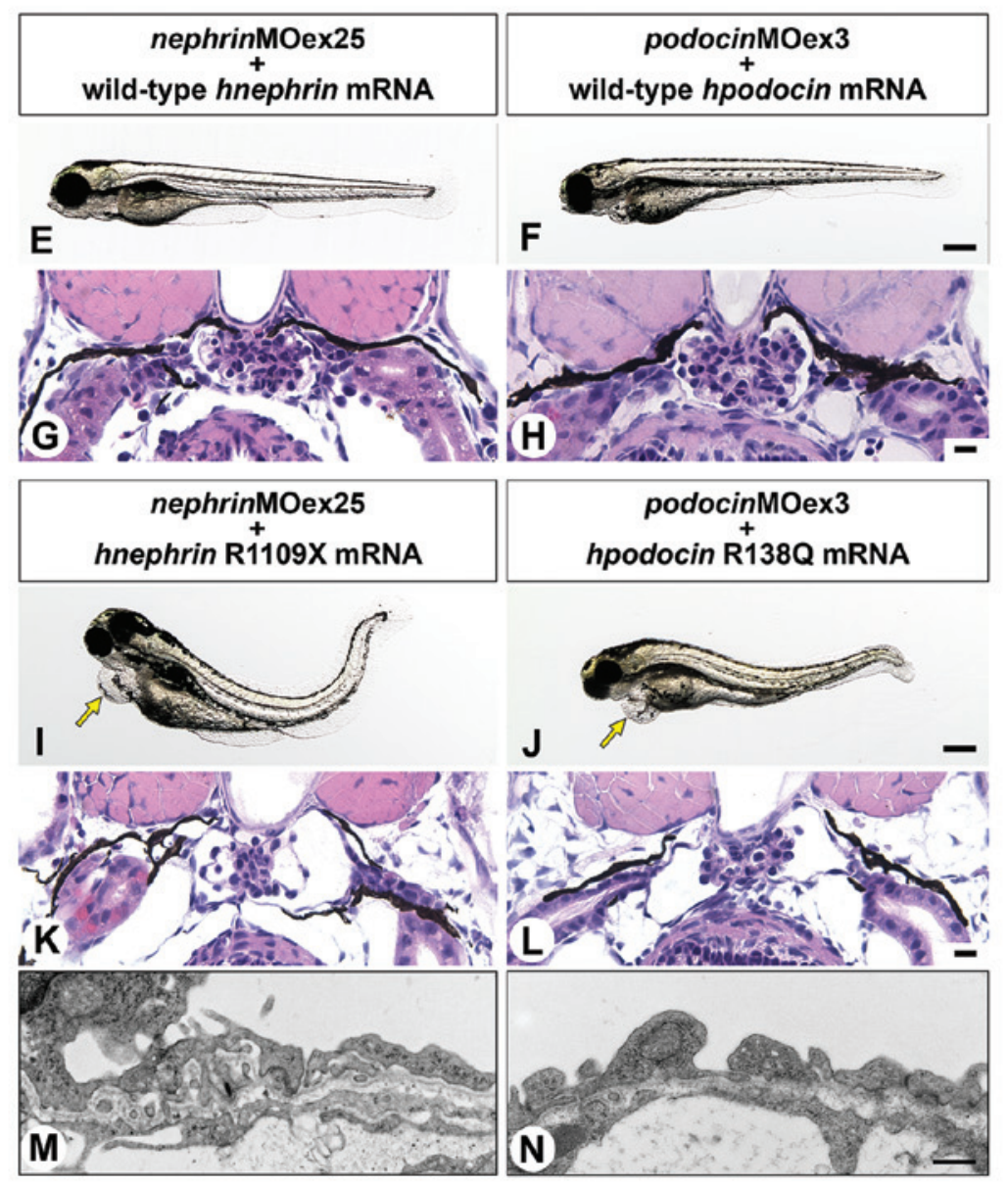

Figure 4. Human mRNA injection. (A) The mutation sites introduced in the human Nephrin and Podocin mRNAs. (B and C) Three types of injected human mRNA (wild-type, NEPHRIN-R1109X and PODOCIN-R138Q) are detected in 2 dpf larvae by RT-PCR. (D) Wild-type mRNAs of human Nephrin and Podocin may rescue $\sim 89 \%$ of nephrinMOex 25 and podocinMOex3 morphants, respectively, however, the percentage of rescued larvae is significantly reduced when mutated mRNA is co-injected at 39 and $8.1 \%$, respectively. The phenotype was scored by pericardial edema, body-axis curvature, glomerulus morphology and altered glomerular barrier. (E-H) Wild-type human Nephrin and Podocin mRNA rescue the morphant phenotypes. (E and F) The larvae exhibit a straight body-axis with no or slight pericardial edema, $(\mathrm{G}$ and $\mathrm{H}$ ) and a well-developed glomerulus. (I-N) Mutated human NEPHRIN-R1109X and PODOCIN-R138Q mRNA do not rescue the morphant phenotypes as frequently. (I and J) Larvae injected with mutated mRNA exhibit pericardial edema and body-axis curvature, (K and L) hypoplastic glomerulus (M and N) and no regular foot processes. Bar scales, $250 \mu \mathrm{m}$ in (F), (J); $10 \mu \mathrm{m}$ in $(\mathrm{H})$, (L) and $100 \mathrm{~nm}$ in $(\mathrm{N})$. dpf, days post-fertilization.

least four days following injection. nephrinMOex25 and podocinMOex3 morpholino-injected embryos exhibited pericardial edema, yolk edema and body-axis curvature. Moreover, the glomerulus was smaller in size compared with the wild-type larvae and contained poorly developed glomerular capillaries and mesangium (Fig. 2F-H). All these features may be rescued by $\sim 89 \%$ by co-injection of human Nephrin and Podocin
mRNAs (Fig. 4D). In addition, rescued morphants exhibited a straight body axis (Fig. 4E and F) and a well-developed pronephric glomerulus at $4 \mathrm{dpf}$ (Fig. 4G and $\mathrm{H}$ ), although slight pericardial edema was observed in the larvae injected with human PODOCIN mRNA and podocinMOex3 (Fig. 4F). These data therefore indicate that the functions of human and zebrafish Nephrin and Podocin are interchangeable. 

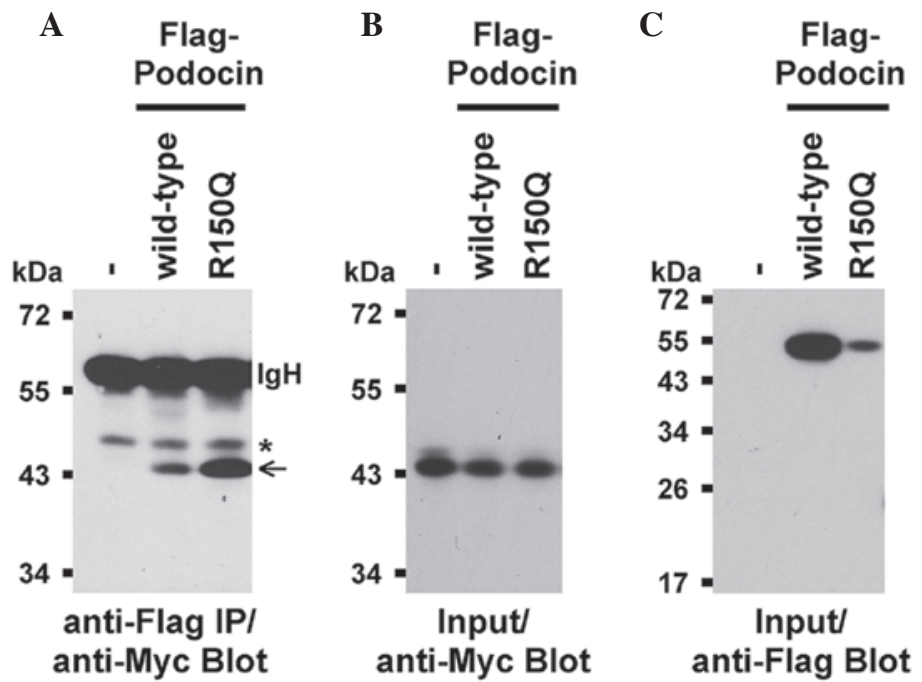

Figure 5. (A) Co-immunoprecipitation assay for Myc-Nephrin and Flag-Podocin. Following anti-Flag IP, coprecipitated Nephrin was revealed by anti-Myc immuno-blotting (see arrow). Asterisk marks a non-specific protein band. IgH, immunoglobulin heavy chain. (B) Similar input levels of Myc-Nephrin. (C) Expression level of wild-type and Flag-tagged Podocin is notably higher in comparison with that of the R150Q mutant. Myc-Nephrin, Myc-tagged zebrafish Nephrin intracelluar region; Flag-Podocin, Flag-tagged zebrafish full-length Podocin; IP, immunoprecipitation.

The phenotype induced by a nephrinMOex25 or podocinMOex3 in zebrafish embryos may be rescued by wild-type human NEPHRIN or PODOCIN but not by NEPHRIN-R1109X and PODOCIN-R138Q mutant mRNA. A number of types of disease-causing mutations in Nephrin and Podocin proteins have been reported in human patients, including R1109X in Nephrin (25) and R138Q in Podocin (26). NEPHRIN-R1109X is a non-sense mutation leading to a large deletion of the intracellular domain of Nephrin and it is one of the two major disease-causing mutations in Finnish-type congenital nephrotic syndrome (24) (Fig. 4A). PODOCIN-R138Q is a missense mutation resulting in the substitution of arginine at position 138 for the uncharged amino acid glutamine and is also one of the most common disease-causing mutations in steroid-resistant nephrotic syndrome (23) (Fig. 4A).

To determine the effect of mutated mRNA on the rescue of the morphant phenotypes, nephrinMOex 25 and podocinMOex3 were injected together with mutated mRNA encoding human NEPHRIN-R1109X and PODOCIN-R138Q, respectively, into one-cell stage embryos. The persistence of the two mutated mRNAs was detected in $4 \mathrm{dpf}$ larvae by RT-PCR (Fig. 4B and C), but the ratio of rescued larvae was significantly lower for the mutated mRNAs compared with the wild-type mRNA (Fig. 4D). Non-rescued larvae exhibited pericardial edema, body-axis curvature and hypoplastic glomerulus, as observed in the larvae injected with the morpholino only (Fig. 4G, H, K and L). The formation of regular foot processes was affected in the larvae injected with NEPHRIN-R1109X and nephrinMOex25, as with nephrinMOex25 alone (Fig. 4M). Notably, the larvae injected with PODOCIN-R138Q and podocinMOex3 did not exhibit any regular foot processes with SD (Fig. 4N). However, larvae injected with podocinMOex3 formed foot processes to some extent (Fig. 2K), indicating that PODOCIN-R138Q exacerbated the morphant phenotype observed with podcinMOex3 alone. The phenotype was scored by morphology and altered glomerular barrier and, in contrast to human Nephrin and Podocin mRNAs, co-injection of and nephrinMOex25 and mutant NEPHRIN-R1109X or podocinMOex3 and PODOCIN-R138Q only rescued 39 and $8.1 \%$, respectively.

Protein interaction between Nephrin and Podocin. To examine whether the protein properties of a mutated PODOCIN were biochemically altered, the abilities of Flag-tagged wild-type and mutated (R150Q) zebrafish full-length Podocin to bind to the Myc-tagged C-terminal intracellular domain of zebrafish Nephrin were compared. Notably, the R150Q zebrafish Podocin mutation corresponded to the disease-causing R138Q mutation in human Podocin. Consistent with previous studies for mammalian Nephrin and Podocin $(17,38)$, binding between wild-type zebrafish Podocin and the intracellular region of Nephrin (Fig. 5) was observed. Binding between the mutated Podocin-R150Q and Nephrin (Fig. 5) was also detected. However, mutated Podocin interacted significantly more with Nephrin despite the lower expression levels of mutated Podocin compared with wild-type Podocin (Fig. 5). The latter may suggest that the mutation of Podocin makes it more prone to protein degradation.

\section{Discussion}

In the mammalian kidney, Nephrin and Podocin localize at the SD between the podocyte foot processes and play essential roles in the formation and maintenance of the SD. A previous study of the localization of zebrafish Nephrin during development revealed its predominant localization at the podocytes within the pronephros, which is similar to its localization in the mammalian metanephros (3). Similarly, Podocin was previously identified to localize in two tortuous line segments within the pronephros, which was similar to that of Nephrin, indicating that Podocin also predominantly localizes at the podocytes in the zebrafish pronephros.

NephrinMOex25 and PodocinMOex3 induced the altered splicing of pre-mRNA to greatly attenuate the expression of 
full-length protein products. Nephrin and Podocin were already expressed in the primitive podocytes of glomerular primordia, which had not yet formed foot processes and SD, suggesting that Nephrin and Podocin contribute to the differentiation of primitive podocytes. The attenuated expression of these two proteins is thus likely to affect the appropriate development and maturation of podocytes. In zebrafish and mammals, podocytes are known to produce vascular endothelial growth factor, which contributes to the migration of primitive capillary endothelial cells and the maturation of glomerular capillaries $(27,39)$. Therefore, impairment of podocyte differentiation may subsequently affect the formation and maturation of other glomerular structures (so-called endocapillary region). The aforementioned scenario may explain the underlying mechanism for the glomerular hypoplasia in the morphants examined.

Although identity in amino acid sequence is low in Nephrin and Podocin homologues between zebrafish and humans, the alignment of the functional domains within the proteins is highly conserved $(9,21)$. Notably, human wild-type Nephrin and Podocin mRNAs may rescue the phenotype of the nephrinMOex25 and podocinMOex3 morphants, respectively. Thus, the current data suggest that the protein function of Nephrin and Podocin is highly conserved between zebrafish and humans.

Human Nephrin and Podocin mRNAs containing disease-causative mutations exhibited significantly lower efficiency in rescuing the morphant phenotypes than the wild-type mRNAs. Moreover, the mRNA coding PODOCIN-R138Q mutant prominently affected the formation of regular foot processes interspaced with $\mathrm{SD}$, although the injection of podocinMOex3 alone did not largely disturb the SD formation. These results suggest that the morpholino and mutated mRNA synergistically interfered with the formation of the foot processes interspaced with SD. Therefore, this experimental system offers potential for investigation of the spatiotemporal consequences of gene mutations associated with the nephrotic syndrome in the early phase of glomerular development. In addition, it may be useful to explore the pathogenic mechanisms underlying human congenital nephrotic syndromes.

Multi-protein complexes, including those containing Nephrin and Podocin are formed at the SD and are believed to be crucial in the establishment and maintenance of the SD. The current study demonstrates that an interaction occurred between wild-type Nephrin and Podocin in zebrafish. Nishibori et al (40) reported that the human PODOCIN-R138Q mutant, which corresponds to the zebrafish podocin-R150Q, is trapped in the endoplasmic reticulum and the trapped Podocin is hypothesized to interfere with trafficking of Nephrin (40). The present pull-down assay indicated that the binding activity of zebrafish podocin-R150Q to Nephrin was stronger compared with wild-type Podocin. If the zebrafish Podocin-R150Q is also trapped and accumulated in the endoplasmic reticulum, the mutated Podocin most likely impaired Nephrin trafficking from the endoplasmic reticulum to its proper destination in the SD near the foot processes due to its abnormally strong ability to bind Nephrin. This hypothesis is in agreement with the observation that the formation of regular foot processes and SD was more detrimentally affected in the larvae injected with human PODOCIN-R138Q mRNA plus podocinMOex3 than with the morpholino alone.
The present study highlights the high evolutionary conservation in the function of Podocin and Nephrin from zebrafish to humans and establishes a system by which disease-causing mutations in human PODOCIN and NEPHRIN may be assessed for their biological consequences. This information is likely to facilitate studies to gain an improved understanding of the development of several kidney diseases, including nephrotic syndrome.

\section{Acknowledgements}

The authors would like to thank Drs Iain Drummond and Lawrence Holzman for their kind gifts of the plasmids; Dr Koichiro Ichimura for his technical assistance in electron microscopy; Drs Deborah Garrity, Kristina Wasson-Blader and Hiroyuki Matsumoto for helpful criticism and comments on the manuscript. T.O. acknowledges financial support from the University of Oklahoma Health Sciences Center (OUHSC). T.O. was supported by NIH grants R21-DK069604 and R01-DK078209. This study was supported in part by the Diabetes Histology and Image Acquisition and Analysis Core Facility at OUHSC (NIH, COBRE-1P20RR024215) and the Imaging Core Facility at the Oklahoma Medical Research Foundation.

\section{References}

1. Ichimura K, Bubenshchikova E, Powell R, Fukuyo Y, Nakamura T, Tran U, Oda, S, Tanaka M, Wessely O, Kurihara H, Sakai T and Obara T: A comparative analysis of glomerulus development in the pronephros of medaka and zebrafish. PloS One 7: e45286, 2012.

2. Ichimura K, Fukuyo Y, Nakamura T, Powell R, Sakai T and Obara T: Structural disorganization of pronephric glomerulus in zebrafish mpp5a/nagie oko mutant. Dev Dyn 241: 1922-1932, 2012.

3. Ichimura K, Fukuyo Y, Nakamura T, Powell R, Sakai T, Janknecht $R$ and Obara T: Developmental localization of nephrin in zebrafish and medaka pronephric glomerulus. J Histochem Cytochem 61: 313-324, 2013.

4. Roselli S, Gribouval O, Boute N, Sich M, Benessy F, Attié T, Gubler MC and Antignac C: Podocin localizes in the kidney to the slit diaphragm area. Am J Pathol 160: 131-139, 2002.

5. Kriz W and Kaissling B: Structural Organization of the Mammalian Kidney. Physiology and pathophysiology. In: Seldin and Giebisch's The Kidney. Alpern RJ, Hebert SC (eds). 4th edition. Academic Press, Waltham, MA, pp. 479-563, 2007.

6. Takahashi-Iwanaga $\mathrm{H}$ : Comparative anatomy of the podocyte: A scanning electron microscopic study. Microsc Res Tech 57: 196-202, 2002.

7. Ichimura K, Kurihara $\mathrm{H}$ and Sakai T: Actin filament organization of foot processes in vertebrate glomerular podocytes. Cell Tissue Res 329: 541-557, 2007.

8. Ichimura K, Kurihara H and Sakai T: Beta-cytoplasmic actin localization in vertebrate glomerular podocytes. Arch Histol Cytol 72: 165-174, 2009.

9. Kramer-Zucker AG, Wiessner S, Jensen AM and Drummond IA: Organization of the pronephric filtration apparatus in zebrafish requires Nephrin, Podocin and the FERM domain protein Mosaic eyes. Dev Biol 285: 316-329, 2005.

10. Fukasawa H, Bornheimer S, Kudlicka K and Farquhar MG: Slit diaphragms contain tight junction proteins. J Am Soc Nephrol 20: 1491-1503, 2009

11. Pavenstädt H, Kriz W and Kretzler M: Cell biology of the glomerular podocyte. Physiol Rev 83: 253-307, 2003.

12. Donoviel DB, Freed DD, Vogel H, Potter DG, Hawkins E, Barrish JP, Mathur BN, Turner CA, Geske R, Montgomery CA, Starbuck M, Brandt M, Gupta A, Ramirez-Solis R, Zambrowicz BP and Powell DR: Proteinuria and perinatal lethality in mice lacking NEPH1, a novel protein with homology to NEPHRIN. Mol Cell Biol 21: 4829-4836, 2001. 
13. Neumann-Haefelin E, Kramer-Zucker A, Slanchev K, Hartleben B, Noutsou F, Martin K, Wanner N, Ritter A, Gödel M, Pagel P, Fu X, Müller A, Baumeister R, Walz G and Huber TB: A model organism approach: defining the role of Neph proteins as regulators of neuron and kidney morphogenesis. Hum Mol Genet 19: 2347-2359, 2010.

14. Dustin ML, Olszowy MW, Holdorf AD, Li J, Bromley S, Desai N, Widder P, Rosenberger F, van der Merwe PA, Allen PM and Shaw AS: A novel adaptor protein orchestrates receptor patterning and cytoskeletal polarity in T-cell contacts. Cell 94: 667-677, 1998.

15. Shih NY, Li J, Karpitskii V, Nguyen A, Dustin ML, Kanagawa O, Miner JH and Shaw AS: Congenital nephrotic syndrome in mice lacking CD2-associated protein. Science 286: 312-315, 1999

16. Shih NY, Li J, Cotran R, Mundel P, Miner JH and Shaw AS: $\mathrm{CD} 2 \mathrm{AP}$ localizes to the slit diaphragm and binds to nephrin via a novel C-terminal domain. Am J Pathol 159: 2303-2308, 2001.

17. Schwarz K, Simons M, Reiser J, Saleem MA, Faul C, Kriz W, Shaw AS, Holzman LB and Mundel P: Podocin, a raft-associated component of the glomerular slit diaphragm, interacts with CD2AP and nephrin. J Clin Invest 108: 1621-1629, 2001.

18. Ruotsalainen V, Ljungberg P, Wartiovaara J, Lenkkeri U, Kestilä M, Jalanko H, Holmberg C and Tryggvason K: Nephrin is specifically located at the slit diaphragm of glomerular podocytes. Proc Natl Acad Sci USA 96: 7962-7967, 1999.

19. Holthofer H, Ahola H, Solin ML, Wang S, Palmen T, Luimula P, Miettinen A and Kerjaschki D: Nephrin localizes at the podocyte filtration slit area and is characteristically spliced in the human kidney. Am J Pathol 155: 1681-1687, 1999.

20. Holzman LB, St John PL, Kovari IA, Verma R, Holthofer H and Abrahamson DR: Nephrin localizes to the slit pore of the glomerular epithelial cell. Kidney Int 56: 1481-1491, 1999.

21. Ebarasi L, He L, Hultenby K, Takemoto M, Betsholtz C, Tryggvason $\mathrm{K}$ and Majumdar A: A reverse genetic screen in the zebrafish identifies crb2b as a regulator of the glomerular filtration barrier. Dev Biol 334: 1-9, 2009.

22. Hentschel DM, Mengel M, Boehme L, Liebsch F, Albertin C, Bonventre JV, Haller $\mathrm{H}$ and Schiffer M: Rapid screening of glomerular slit diaphragm integrity in larval zebrafish. Am J Physiol Renal Physiol 293: F1746-F1750, 2007.

23. Boute N, Gribouval O, Roselli S, Benessy F, Lee $\mathrm{H}$, Fuchshuber A, Dahan K, Gubler MC, Niaudet P and Antigna C: NPHS2, encoding the glomerular protein podocin, is mutated in autosomal recessive steroid-resistant nephrotic syndrome. Nat Genet 24: 349-354, 2000

24. Kestilä M, Lenkkeri U, Männikkö M, Lamerdin J, McCready P, Putaala H, Ruotsalainen V, Morita T, Nissinen M, Herva R Kashtan CE, Peltonen L, Holmberg C, Olsen A and Tryggvason K: Positionally cloned gene for a novel glomerular protein - nephrin - is mutated in congenital nephrotic syndrome. Mol Cell 1: 575-582, 1998

25. Beltcheva O, Martin P, Lenkkeri U and Tryggvason K: Mutation spectrum in the nephrin gene (NPHS1) in congenital nephrotic syndrome. Hum Mutat 17: 368-373, 2001.
26. Caridi G, Perfumo F and Ghiggeri GM: NPHS2 (Podocin) mutations in nephrotic syndrome. Clinical spectrum and fine mechanisms. Pediatr Res 57: 54R-61R, 2005.

27. O'Brien LL, Grimaldi M, Kostun Z, Wingert RA, Selleck R and Davidson AJ: Wt1a, Foxcla, and the Notch mediator Rbpj physically interact and regulate the formation of podocytes in zebrafish. Dev Biol 358: 318-330, 2011.

28. Thisse $\mathrm{C}$ and Thisse B: High-resolution in situ hybridization to whole-mount zebrafish embryos. Nat Protoc 3: 59-69, 2008.

29. Link V, Shevchenko A and Heisenberg CP: Proteomics of early zebrafish embryos. BMC Dev Biol 6: 1, 2006.

30. Wu J and Janknecht R: Regulation of the ETS transcription factor ER81 by the 90-kDa ribosomal S6 kinase 1 and protein kinase A. J Biol Chem 277: 42669-42679, 2002.

31. Shin S and Janknecht R: Diversity within the JMJD2 histone demethylase family. Biochem Biophys Res Commun 353: 973-977, 2007.

32. Papoutsopoulou S and Janknecht R: Phosphorylation of ETS transcription factor ER81 in a complex with its coactivators CREB-binding protein and p300. Mol Cell Biol 20: 7300-7310, 2000.

33. Bubenshchikova E, Ichimura K, Fukuyo Y, Powell R, Hsu C, Morrical SO, Sedor JR, Sakai T and Obara T: Wtip and Vangl2 are required for mitotic spindle orientation and cloaca morphogenesis. Biol Open 1: 588-596, 2012

34. Knebel J, De Haro L and Janknecht R: Repression of transcription by TSGA/Jmjdla, a novel interaction partner of the ETS protein ER71. J Cell Biochem 99: 319-329, 2006.

35. Kim TD, Shin S and Janknecht R: Repression of Smad3 activity by histone demethylase SMCX/JARID1C. Biochem Biophys Res Commun 366: 563-567, 2008

36. Ruotsalainen V, Patrakka J, Tissari P, Reponen P, Hess M, Kestilä M,Holmberg C, Salonen R, Heikinheim M, Wartiovaara J, Tryggvason $\mathrm{K}$ and Jalanko H: Role of nephrin in cell junction formation in human nephrogenesis. Am J Pathol 157: 1905-1916, 2000.

37. Kawachi H, Abrahamson DR, St John PL, Goldstein DJ, Shia MA, Matsui K, Shimizu F and Salant DJ: Developmental expression of the nephritogenic antigen of monoclonal antibody 5-1-6. Am J Pathol 147: 823-833, 1995.

38. Huber TB, Simons M, Hartleben B, Sernetz L, Schmidts M, Gundlach E, Saleem MA, Walz G and Benzing T: Molecular basis of the functional podocin-nephrin complex: mutations in the NPHS2 gene disrupt nephrin targeting to lipid raft microdomains. Hum Mol Genet 12: 3397-3405, 2003.

39. Kitamoto $\mathrm{Y}$, Tokunaga $\mathrm{H}$ and Tomita $\mathrm{K}$ : Vascular endothelial growth factor is an essential molecule for mouse kidney development: glomerulogenesis and nephrogenesis. J Clin Invest 99: 2351-2357, 1997.

40. Nishibori Y, Liu L, Hosoyamada M, Endou H, Kudo A, Takenaka H, Higashihara E, Bessho F, Takahashi S, Kershaw D, Ruotsalainen V, Tryggvason K, Khoshnoodi J and Yan K: Disease-causing missense mutations in NPHS2 gene alter normal nephrin trafficking to the plasma membrane. Kidney Int 66: 1755-1765, 2004 